\title{
Decision optimization for face recognition based on an alternate correlation plane quantification metric
}

\author{
A. Alfalou, ${ }^{1,4}$ C. Brosseau, ${ }^{2, *}$ P. Katz, ${ }^{1}$ and M. S. Alam ${ }^{3}$ \\ ${ }^{1}$ ISEN Brest, Département Optoélectronique, L@bISEN, 20 rue Cuirassé Bretagne, CS 42807, 29228 Brest Cedex 2, France \\ ${ }^{2}$ Université Européenne de Bretagne, Université de Brest, Lab-STICC, CS 93837, 6 avenue Le Gorgeu, 29238 Brest Cedex 3, France \\ ${ }^{3}$ Department of Electrical and Computer Engineering University of South Alabama, 6001 USA South Dr., Mobile, AL 36688-0002, USA \\ ${ }^{4}$ e-mail: ayman.al-falou@isen.fr \\ ${ }^{*}$ Corresponding author: brosseau@univ-brest.fr
}

Received December 12, 2011; revised January 31, 2012; accepted February 11, 2012;

posted February 13, 2012 (Doc. ID 159824); published May 2, 2012

\begin{abstract}
We consider a new approach for enhancing the discrimination performance of the VanderLugt correlator. Instead of trying to optimize the correlation filter, or propose a new decision correlation peak detection criterion, we propose herein to denoise the correlation plane before applying the peak-to-correlation energy (PCE) criterion. For that purpose, we use a linear functional model to express a given correlation plane as a linear combination of the correlation peak, noise, and residual components. The correlation peak is modeled using an orthonormalized function and the singular value decomposition method. A set of training correlation planes is then selected to create the correlation noise components. Finally, an optimized correlation plane is reconstructed while discarding the noise components. Independently of the filter correlation used, this technique denoises the correlation plane by lowering the correlation noise magnitude in case of true correlation and decreases the false alarm rate when the target image does not belong to the desired class. Test results are presented, using a composite filter and a face recognition application, to verify the effectiveness of the proposed technique. (C) 2012 Optical Society of America
\end{abstract}

OCIS codes: $100.5010,100.3008$.

During the last decade, much work has been done in the realm of image recognition techniques. The correlator block diagram shown in Fig. 1 is one of the most promising approaches for these techniques. This diagram consists of an optical part for the correlation process, a filter part, and a decision part. The second and third parts are generally implemented electronically, whereas the first part is usually implemented using an optical setup. To increase the decisional performance of the correlators, much work has been performed by optimizing the correlation filter and/or choosing a specific decision criterion, e.g. signal-to-noise ratio (SNR), and PCE [1-8]. Based on a new concept of correlation filter, we recently suggested an optimization of the correlation plane for a face recognition application [5].

However, this optimization method is limited by the interpretation of the correlation plane. In addition, processing is carried out offline. Here, we propose to apply a postprocessing step in the correlation plane before using a decision criterion. Our specific aim is to increase the correlation peak characteristics when the test image belongs to a predefined class, and decrease the false alarm rate when the target image does not belong to this class.

In this letter, we achieve this by introducing an alternate correlation plane quantification metric, based on the linear functional model (LFM) [8-9] and singular value decomposition (SVD) method $[\underline{10}]$. The main advantage of our method lies in its ability to cancel out the background correlation plane (i.e., to denoise the correlation plane) which has for effect to discriminate the correlation peak. Indeed, we assumed that the signal and noise are mutually independent in the correlation plane (input plane of the proposed algorithm). That is, the correlation plane is considered as a combination of the desired signal and background noise. The proposed technique is simple to implement offline and it produces a higher percentage of true class matches in comparison to traditional techniques; i.e., the true positive rate (TPR) is increased by a factor of five for a false positive rate (FPR) set to $0 \%$. After presenting technical aspects of the LFM-SVD algorithm, we present simulation results that illustrate the performance of the proposed technique using the Pointing Head Pose Image Database (PHPID) [11].

The principle of our algorithm is shown in Fig. 2. At first, the input correlation plane $P_{c}$ [Fig. 2(a)] is decomposed as a linear combination of different regressors. We model the signal and noise regressors by using respectively a set of false correlation planes $Y^{\text {noise }}$ [Fig. 2(b)], and a set of sine cardinal functions with different characteristics $Y^{\text {peak }}$ [Fig. 2(c)]. Secondly, these regressors are used to describe the input plane with a linear combination of peak, noise components, and a residual signal. Finally, the output correlation plane is reconstructed without the noise components [Fig. 2(d)]. An important advantage of this method is that it gives a well defined and sharp peak for a true correlation and a negligible peak for false correlation.

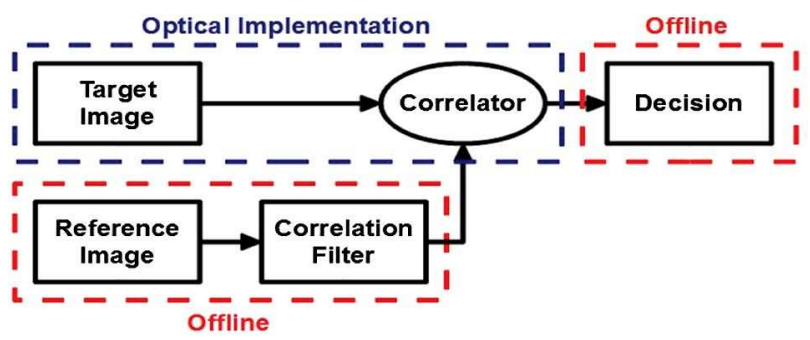

Fig. 1. (Color online) Illustrating the principle of correlation. 
(b)

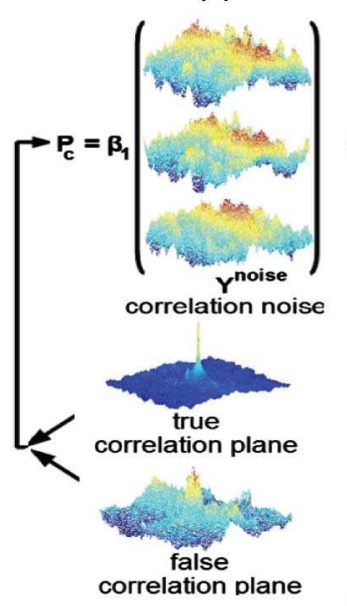

(a)

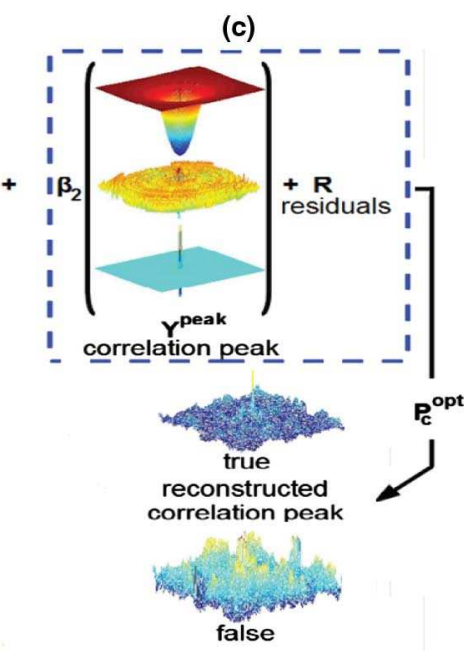

(d)

Fig. 2. (Color online) Synoptic diagram of the correlation plane denoising process.

To put this more precisely, the starting point is to set the correlation plane $P_{c}$ equal to a finite linear combination of weighted regressors, expressed as

$$
P_{c}=\sum_{i=1}^{M} \beta_{i} Y_{i}+R
$$

where $M$ is a given integer number, $\beta_{i}$ denotes the weight corresponding to regressor $Y_{i}=Y_{i}^{\text {noise }}+Y_{i}^{\text {peak }}$, and $R$ is a residual signal. In practice, it is convenient to rewrite Eq. (1) in a matrix form, given by $P_{c}=Y \beta+R$, which defines the optimal unbiased estimator $\beta$ assuming that the residual noise is white, and using the Moore-Penrose pseudoinverse of $\boldsymbol{Y}$. Eq. (1) shows that the decomposition of the correlation plane depends on the regressors; i.e., a sum of weighted regressors is generated to ensure the best possible fit of the real correlation plane. Thus, the input correlation plane (Fig. 2) resulting from the correlation process, called $P_{c}$ is decomposed into a linear combination of regressors; i.e., correlation noise, correlation peak components, and a residual signal, respectively $Y^{\text {noise }}, Y^{\text {peak }}$, and $R$. As the aim of our algorithm is to reduce the background correlation plane in order to obtain sharper peaks for a match, the signal is then reconstructed by totally discarding the noise components used in the decomposition process, thereby resulting in an optimized correlation plane, $P_{c}^{\text {opt }}$. The estimation problem amounts to finding specific regressors for both signal and noise [9]. Denoising the correlation plane $\left[P_{c}^{\text {opt }}\right.$, Fig. $\left.\underline{2(\mathrm{~d})]}\right]$ consists in retaining only the desired information (information regressors $Y^{\text {peak }}$ ) and removing noise information (noise regressors $Y^{\text {noise }}$ Fig. 2). Prior to defining the regressors for the information signal, we approximate the correlation peak by a threedimensional sine cardinal function, expressed as

$$
\operatorname{Peak}(i, j)=\left|\sin c\left(\frac{\left(i-i_{0}\right)^{2}}{2 \sigma_{i}^{2}}\right)+\sin c\left(\frac{\left(j-j_{0}\right)^{2}}{2 \sigma_{j}^{2}}\right)\right|
$$

where Peak $(i, j)$ defines the correlation peak value at $(i, j)$ pixel in the output plane, sin $c(x)$ is the sine cardinal function, and $\sigma_{i}$ and $\sigma_{j}$ denote the standard deviations for $i$ and $j$ around their respective mean values $i_{0}$ and $j_{0}$. The parameters in Eq. (2) were varied in order to get a large number of correlation planes $(k)$ characterized with different correlation peak shapes Peak 1, Peak $_{2}, \ldots$ Peak $_{k}$. Then, we expand these simulated correlation planes in terms of a set of orthonormal vectors. This is done by using the thin SVD matrix factorization to obtain the right singular vectors $V_{t}[\underline{10}]$. The regressors $Y^{\text {peak }}$ are given by

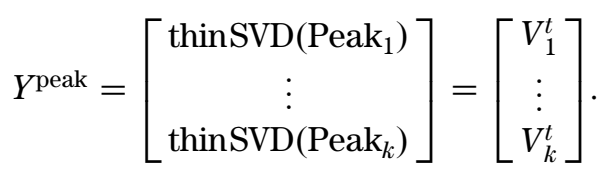

The next step is to model the correlation plane noise. At first, we generate a face database for modeling the noise. Four subjects from the PHPID dataset were selected for this purpose, as shown in Fig. 3(a). Next, 52 facial images per person $\mathrm{P}(\mathrm{j})$, were obtained by rotating from right to left and from top to bottom. These images were correlated with a phase-only filter (POF: the chosen filter to validate the principle of our algorithm) formulated by choosing the face of the subject $\mathrm{P}(1)$ as shown in Fig. 3(a). Each of these correlations yields a noise realization noise $l(1 \leq l \leq n)$, where $n$ denotes the number of noise models. In order to model the noise associated with subject $\mathrm{P}(1)$, the information signal (correlation peak) is suppressed while keeping the noise signal undisturbed. The full correlation plane is considered for the other faces. Using this analysis, we obtain the noise column vector, denoted as

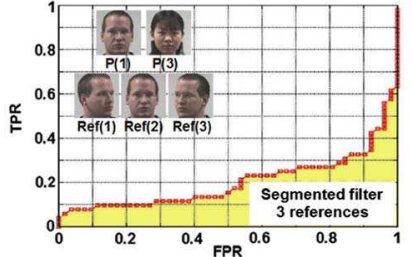

(a)

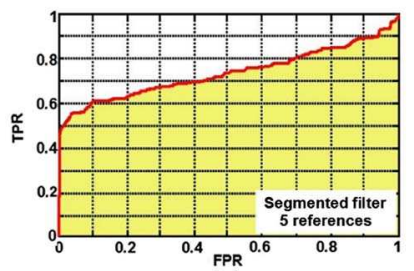

(c)

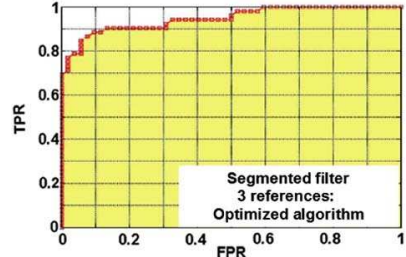

(b)

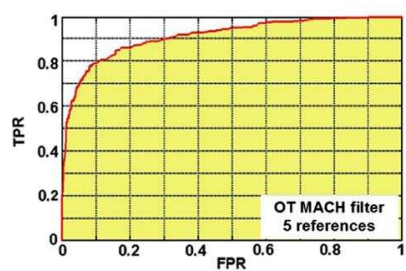

(d)
Fig. 3. (Color online) The results obtained using different kinds of filters. The first row shows the ROC curves using a 3-reference segmented filter: (a) without optimization, (b) with our optimized method and using 12 correlation plane noise models. The second row shows the corresponding results using 5-references filters: (c) using a segmented composite filter, (d) using a OT MACH filter. 


$$
\boldsymbol{Y}^{\text {noise }}=\left[\begin{array}{c}
\text { noise }_{1} \\
\vdots \\
\text { noise }_{n}
\end{array}\right] .
$$

Using Eqs. (3) and (י), $\boldsymbol{Y}$ can be expressed as

$$
\boldsymbol{Y}=\left[\begin{array}{c}
\boldsymbol{Y}^{\text {peak }} \\
\boldsymbol{Y}^{\text {noise }}
\end{array}\right] .
$$

Now, the target correlation plane is decomposed thanks to the definition of the linear model of Eq. (1), without residual noise; i.e., weights corresponding to different types of regressors are calculated. The reconstructed correlation plane can be expressed as $\boldsymbol{P}_{c}^{\prime}=\boldsymbol{Y} \boldsymbol{\beta}^{+}$, where $P_{c}^{\prime}$ represents the reconstructed correlation plane and $\beta^{+}$represents the weights of the regressors. The residuals are obtained by calculating the difference between the target correlation plane $P_{c}$ and the reconstructed correlation plane $P_{c}^{\prime}$; i.e., $\boldsymbol{R}=\boldsymbol{P}_{c}-\boldsymbol{P}_{c}^{\prime}$. Hence, the optimized correlation plane can be expressed by $\boldsymbol{P}_{c}^{\text {opt }}=$ $\boldsymbol{Y}^{\text {peak }} \boldsymbol{\beta}_{\text {peak }}^{+}+\boldsymbol{R}$, where $\beta_{\text {peak }}^{+}$defines the weights of the regressors for the signal $Y^{\text {peak }}$.

The optimized correlation plane performance measure is the PCE. As will be shown shortly, one important advantage of this procedure is to lower the false alarm rate significantly. To illustrate the robustness of the proposed technique for face recognition, we tested it with a VanderLugt correlator using a 3-reference segmented filter [1] generated using the facial images of [1-3] as shown in Fig. 3(a).

Correlating subject $\mathrm{P}(1)$ with the segmented filter yields a true correlation, whereas correlating subject $\mathrm{P}$ (3) with this filter yields a false alarm. Fig. 3(a) shows the results obtained by correlating the 52 images of subject $\mathrm{P}(1)$ and subject $\mathrm{P}(3)$ with the 3-reference segmented filter. Fig. 2(b) represents the ROC curves obtained with 12 models for each subject with optimization. The reconstruction of the output correlation plane requires a processing time of $0.0883 \mathrm{~s}$ running Windows 7 (64bit), $3.1 \mathrm{GHz}$ CPU 4 GB RAM with the MATLAB software package. For comparison, Fig. 3(c) and Fig. 3(d) represent the results from the 5-reference filters using a segmented filter and an OT MACH filter, respectively [12]. From Fig. $\underline{3}$, we can see that without optimization, the TPR is close to $15 \%$ for a FPR set to $0 \%$. Using the proposed technique, the VLC generates a TPR close to $70 \%$ of correct face identification with zero false positives. This recognition rate value exceeds the corresponding value for the optimized filter in [5]. To confirm the superior performance of our optimization, we compared it with an optimized 5-reference filter that yields a TPR close to $50 \%$ and $30 \%$, respectively for the segmented and the trade-off maximum average correlation height (OT-
$\mathrm{MACH}$ ) filters. For both cases we found that the recognition rate was largely above $70 \%$. As a benchmark for correlation plane quantification, it is instructive to compare the corresponding value of the area under curve (AUC) without optimization [Fig 3(a), which is equal to 0.201] and that obtained using our methodology [Fig. 3(b), which is 0.947] which is significantly larger.

Overall, our results dealing with different subjects indicate that the recognition rate tends to increase as the number of noise and peak models increases. To summarize, we have proposed a new LFM-SVD-based recognition algorithm for improved face recognition via correlation plane optimization. Test results obtained using this algorithm clearly indicate the effectiveness of this approach in relation to face recognition [4]. We have achieved recognition rates around $70 \%$ with $100 \%$ discrimination with the PHPID database. Our findings suggest that this algorithm can be adapted for different decision criteria and optimized correlation filters. Various tests show that it is possible to increase the robustness of the correlator while keeping a very good discrimination quality, typically comparable to that of the amplitude modulated phase only matched filter [3] . Furthermore, the simplicity of the proposed technique makes it is easier to implement. Future work may include investigation with other composite filters to show that this method is not segmented filter specific.

\section{References}

1. A. Alfalou and C. Brosseau, in Face Recognition, Milos Oravec, ed. (INTECH, 2010), pp. 354-380.

2. F. T. S. Yu and S. Jutamulia, Optical Pattern Recognition (Cambridge University, 1998).

3. A. A. S. Awwal, Appl. Opt. 49, B40 (2010).

4. P. Katz, A. Alfalou, C. Brosseau, and M. S. Alam, "Correlation and independent component analysis based approaches for biometric recognition," in Face Recognition: Methods, Applications and Technology, (INTECH, to be published).

5. A. Alfalou and C. Brosseau, Opt. Lett. 36, 645 (2011).

6. F. Dubois, Appl. Opt. 35, 4589 (1996).

7. R. D. Juday, J. Opt. Soc. Am. A 18, 1882 (2001).

8. H. Cardot, F. Ferraty, and P. Sarda, Statist. Probab. Lett. 45, 11 (1999).

9. A. Reynaud, S. Takerkart, G. S. Masson, and F. Chavane, NeuroImage 54, 1196 (2011).

10. M. Wall, A. Rechtsteiner, and L. Rocha, in A Practical Approach to Microarray Data Analysis, D. P. Berrar, W. Dubitzky, and M. Granzow, eds. (Springer, 2003), pp. 91-109.

11. N. Gourier, D. Hall, and J. L. Crowley, in Proceedings of Pointing 2004, ICPR, International Workshop on Visual Observation of Deictic Gestures (2004).

12. H. Zhou and T. H. Chao, Proc. SPIE 3715, 394 (1999). 